\title{
Degrees of belief, expected and actual
}

\author{
Rosanna Keefe ${ }^{1}$
}

Received: 12 June 2014 / Accepted: 12 February 2016 / Published online: 1 March 2016

(C) The Author(s) 2016. This article is published with open access at Springerlink.com

\begin{abstract}
A framework of degrees of belief, or credences, is often advocated to model our uncertainty about how things are or will turn out. It has also been employed in relation to the kind of uncertainty or indefiniteness that arises due to vagueness, such as when we consider " $a$ is F" in a case where $a$ is borderline F. How should we understand degrees of belief when we take into account both these phenomena? Can the right kind of theory of the semantics of vagueness help us answer this? Nicholas J.J. Smith defends a unified account, according to which "degree of belief is expected truth-value"; this builds on his Degree Theory of vagueness that offers an account of the semantics and logic of vagueness in terms of degrees of truth. I argue that his account fails. Degree theories of vagueness do not help us understand degrees of belief and, I argue, we shouldn't expect a theory of vagueness to yield a detailed uniform story about this. The route from the semantics to psychological states needn't be straightforward or uniform even before we attempt to combine vagueness with probabilistic uncertainty.
\end{abstract}

Keywords Degrees of belief - Degree theories of vagueness - Credence - Vagueness Uncertainty $\cdot$ Supervaluationism $\cdot$ N. J. J. Smith

\section{Frameworks of degrees}

In many cases when I am inclined to say I believe that p, I am not certain of p's truth. Perhaps I have good evidence that $\mathrm{p}$, but insufficient evidence for certainty. In some cases-perhaps a claim about the future where I can predict, without complete

Rosanna Keefe

r.keefe@sheffield.ac.uk

1 Department of Philosophy, University of Sheffield, 45 Victoria Street, Sheffield S3 7QB, England 
confidence, how things will turn out - I may want to say that I believe "probably p". In other cases, I may be not inclined to claim to believe p, not because I think that not-p, but because I'm not sure whether or not p. All such cases, among others, have been dealt with using a framework of degrees of belief, or credences. Degrees of belief-or credences - range from 0 to 1 , with a degree of belief of 1 amounting to certainty and 0 to certainty of the negation. In cases where I'm generally happy to assert $p$, but, if pushed, will acknowledge that I'm not certain that $\mathrm{p}$, I can be described as having a degree of belief less than, if relatively close to, 1 . Cases where I want to say I'm not sure whether or not $\mathrm{p}$, can amount to different degrees of belief that $\mathrm{p}$ according to the extent to which I, loosely speaking, am confident that p. We can think of someone's set of beliefs as an assignment of such values to propositions. There are, then, rational constraints on the relations between the degree of belief in a compound and in their components. For example, your degree of belief in a conjunction should (and, if you are rational, will) be no greater than the degree of belief in each of the conjuncts. The standard framework for modelling these relations is a probabilistic one.

Suppose you are considering the proposition that $a$ is $\mathrm{F}$, for some predicate $\mathrm{F}$, while knowing all the relevant information about $a$ that relates to whether $a$ counts as $\mathrm{F}$ for example, you are considering "Tek is tall", while knowing Tek's height. ${ }^{1}$ If $a$ is borderline F, you may still feel a kind of uncertainty about " $a$ is F". Asked whether you believe that Tek is tall, when Tek is borderline tall, you may resist saying yes or no and be inclined to report some attitude between belief and disbelief. Arguably, such attitudes should also be modelled as degrees of belief. If Tim is taller than Tek, but still borderline tall, I will have an intermediate degree of belief in "Tim is tall" that is bigger than my degree of belief in "Tek is tall". If Todd is an absolutely definite case of $\mathrm{F}$ (e.g. someone with no hair is definitely bald), then I should have a degree of belief of 1 in the proposition that Todd is F. Degrees of belief could then be seen as also resulting from vagueness-e.g. the vagueness of our classificatory term "tall"-and the indeterminacy that borderline cases yield. We may expect there again to be rational relations between degrees of belief in compounds and in their vague components; as we will see later, those who focus on degrees within a theory of vagueness typically advocate a non-probabilistic degree-theoretic structure.

If vagueness can yield degrees of belief and so can uncertainty, what picture should we expect when we consider both these phenomena together? Suppose, for example, that I've never seen Tom, who is the fully-grown biological son of two old friends, I may have some expectation as to his height (by knowing the height of his parents), and it may seem likely that he is borderline tall. My degree of belief in "Tom is tall" will be affected by both my expectation of his likely height and the vagueness over whether certain likely heights should count as "tall". How should these factors interact? The problems with combining the phenomena may seem particularly acute if we think that the structures of degrees are different for the two phenomena (probabilistic in one case and not in the other). If we hope to deal with both alleged sources of degrees of belief, we may hope to be able to offer a unified account that explains the way in which uncertainty and vagueness can combine to determine the degree of belief

1 I put aside contextual variation here: let's suppose you know all the relevant facts about the comparison class too. 
in a proposition. This is the approach I will critically focus on in particular in this paper. One alternative to that option is to take the two phenomena to be separate, so that we might simultaneously have each different type of degree of belief in the same proposition, without many constraints on how those different degrees are related. ${ }^{2}$ I will not examine this position here. It should be noted, however, that any arguments against this option do not yet serve as a defence of the first option, without ruling out a third option, which, in the last section, I suggest is the best approach. This third option denies that a systematic degree-theoretic treatment of belief is viable in the vagueness case, in particular denying that a theory of vagueness can yield such an account.

A natural way to unify would be to maintain that there is a single notion of degree of belief and that our degrees of belief can be affected by both uncertainty and vagueness. It may be convenient to explain credence by considering cases where vagueness is not an issue, but perhaps we can smoothly accommodate those cases where vagueness is present. Maybe our intermediate degree of belief in "Tek is tall" just reflects the uncertainty we have in whether he is rightly classified as tall. That uncertainty is compatible with thinking we'll never find out whether he's rightly so classified and, indeed, with assuming there is no fact of the matter about whether he's rightly so classified. In other words, this unified approach may be combined with an epistemic theory of vagueness that maintains that there is an unknown fact of the matter about whether Tek is tall, but also with a semantic theory of vagueness that maintains that there is genuine indeterminacy-propositions that are neither true nor false-in the presence of vagueness. One concern about this unified approach arises from the thought that the degree-theoretic structure arising from the two phenomena is different. To present this objection, it is useful to explain a type of theory of vagueness that employs degrees over and above psychological states of degrees of belief - a Degree Theory of Vagueness.

According to a typical Degree Theory of Vagueness, borderline predications are neither true nor false, but take some intermediate truth-value or degree of truth. ${ }^{3}$ "Tek is tall" may be true to degree 0.4 while "Tim is tall" may be true to degree 0.45 , recalling the taller but still borderline-tall Tim. Take a sorites series of men where each is very slightly less tall (say just one hundredth of inch) than the previous one, starting with a clearly tall man and ending with a clearly not-tall one. The degree of tallness gradually drops through the intermediate cases, as, correspondingly, does the degree of truth of " $x$ is tall". To illuminate the logic governing a language infected by such vagueness and to assess the main premise of the sorites paradox corresponding to such a series - "if $\mathrm{x}$ is taller and $\mathrm{y}$ is one hundredth of an inch shorter, then $\mathrm{y}$ is also tall"-we will need to consider compound sentences. On a degree-functional account, the degree of truth of a compound is determined by the degrees of truth of its components according to the degree-theoretic definition of the relevant connectives (or other logical components). There are a number of options for these definitions, but most commonly, the degree of truth of a conjunction, $\mathrm{P} \& \mathrm{Q}$, is the same as the lowest of the degrees of $\mathrm{P}$ and of $\mathrm{Q}$, while the degree of a disjunction $\mathrm{P} \vee \mathrm{Q}$ is the highest of

\footnotetext{
2 See, e.g., Schiffer (2000).

3 E.g. Smith (2008). See also, e.g., Keefe (2000, Chap. 4), for a summary of other views of this type.
} 
those two degrees. ${ }^{4}$ This degree-functional story thus diverges from the probabilistic ones: the probability of $\mathrm{P} \& \mathrm{Q}$ is not determined just by the probability of $\mathrm{P}$ and the probability of $\mathrm{Q}$, since it is also affected by the relations between $\mathrm{P}$ and $\mathrm{Q}$.

A degree theory of vagueness of this kind could be conveniently co-opted into a story about our degrees of belief if we are focusing on how these degrees are affected by the indeterminacy characteristic of vagueness. If we have intermediate degrees of belief $\mathrm{p}_{1}$ in $\mathrm{P}$ and $\mathrm{p}_{2}$ in $\mathrm{Q}$, our typical, and rational, degree of belief in $\mathrm{P} \& \mathrm{Q}$ will then be the smaller of $p_{1}$ and $p_{2}$, reflecting its truth-value. This, indeed, is the approach taken by a number of advocates of degree theories. They argue, for example, that their treatment of the truth-value of conjunctions successfully reflects our attitudes to conjunctions, in contrast with a probabilistic story about degrees of belief which fails because it treats conjunction differently. Smith summarises the relations as follows: "degrees of belief [arising from vagueness] do not behave in the same ways as degrees of belief arising from uncertainty: they do not conform to the laws of probability" (2010, p.491, see also Schiffer 2000, p. 225). In the next section I turn to the details of one proposal for combining vagueness and uncertainty in the light of these purported structural differences.

\section{Degree of belief is not expected truth value}

Nicholas J. J. Smith's account of degrees of belief attempts to unify the degrees arising from vagueness and uncertainty $(2010,2014)$. Let's start with his "vagueness-free situations" (VFS)—where the relevant predicates determinately apply or determinately don't apply, even if we don't always know which—and "uncertainty-free situations" (UFS), where, roughly, there is vagueness about what predicates apply but no uncertainty about the relevant facts. Given the claims about the structure of degrees arising from the two sources, he promotes the merit of his theory that in a VFS, the structure of our degrees of belief is probabilistic, whereas in an UFS, it fits the non-probabilistic degree-theoretic structure of his theory. When there is both uncertainty and vagueness, both elements impact on degrees of belief. Take the following relatively straightforward toy case. You judge that it is equally likely that Don has height $\mathrm{h}_{1}$ as that Don has height $\mathrm{h}_{2}$ (and no chance he has any other height). You also know that people of $h_{1}$ and $h_{2}$ are borderline tall to degrees $d_{1}$ and $d_{2}$ respectively, (and so you have degree of belief $d_{1}$ in "men of height $h_{1}$ are tall" etc.). What degree of belief do you have that Don is tall, given both these uncertainties and vagueness? Smith's answer averages out the half chance of $d_{1}$ and the half chance of $d_{2}$ and gets a degree of belief of $0.5 \times \mathrm{d}_{1}+0.5 \times \mathrm{d}_{2}$. If you think $\mathrm{h}_{1}$ more likely to be Don's height—say probability 0.8 - the contribution to the combined degree of belief is correspondingly higher: $0.8 \times \mathrm{d}_{1}+0.2 \times \mathrm{d}_{2}$. And so on (generalising for more possibilities etc.), reaching the picture that the degree of belief is the expected truth-value.

Let's sketch the model in a little more detail. Smith employs a function, P, from subsets of possible worlds to real numbers between 0 and 1, which captures the sub-

\footnotetext{
4 I will not focus on the more controversial treatment of the conditional here. A common option is to take the degree of $\mathrm{P} \rightarrow \mathrm{Q}$ to be 1 minus the degree to which $\mathrm{Q}$ falls below $\mathrm{P}$.
} 
ject's "epistemic state" and amounts to a measure of the subject's judgement that the actual world is among that subset of possible worlds. A proposition, S, determines a function S', which maps each world to the degree of truth of the proposition at that world. The proposal is that the subject's degree of belief in $\mathrm{S}$ is the expected value of $\mathrm{S}$, e.g., for the finite case: $\mathrm{P}\left(\left\{\mathrm{w}_{1}\right\}\right) \times \mathrm{S}^{\prime}\left(\mathrm{w}_{1}\right)+\cdots+\mathrm{P}\left(\left\{\mathrm{w}_{\mathrm{n}}\right\}\right) \times \mathrm{S}^{\prime}\left(\mathrm{w}_{\mathrm{n}}\right)$. (See Smith 2010, pp. 496-497.) As desired, in a VFS, each element of the sum will be $P\left(\left\{w_{i}\right\}\right)$ multiplied by 1 or 0 and the sum thus corresponds to the expected probability, with compounds of such values following a probabilistic structure. And in an UFS, the formula will deliver a "sum" with just one element, and the structure of compounds will follow the degree-theoretic structure of degrees of truth in Smith's theory.

This picture cannot succeed in illuminating our degrees of belief however. It allows, relative to each world, for a contribution from borderline cases reflecting the extent to which they are true-their actual truth-values-but thereby takes on an objective feature of the situation rather than the subjective state of the believer in question. The contribution to the sum made in relation to a given world is determined by the subjective judgement of its probability - appropriately — but the objective facts about the degree of truth of the proposition in question in that world. Smith, for example, explains degrees of truth in terms of degrees of closeness (how close Tek is to being tall is reflected in the degree to which he counts as tall). But degrees of belief should correspond to our different judgements of, say, how close a borderline case of F-ness is to being $\mathrm{F}$ which might not be an accurate judgement of degree of closeness.

Whatever one's theory of vagueness, if we want a story about subjective degrees of belief in the light of vagueness, we need to allow for inaccurate subjective states and different states for different people. Bob may be more "generous" with his judgement of tallness than Cam. If forced to guess where the boundary was (or draw a suitable boundary) Bob would place it lower than Cam. And faced with Tek, Bob would be a little more inclined to call him tall than Cam would. It would be natural to say here that Bob's degree of belief in "Tek is tall” is higher than Cam's. This is a matter of a difference in their judgements. It is a difference that you would be bound to find if, for example, the epistemic view were correct. One of Bob and Cam may then be more accurate (so, e.g., will draw the boundary to "tall" closer to the real boundary if forced to draw one and will have a higher degree of belief in "Tek is tall" if that proposition is (unknowably) true). If we reject the epistemic view, the described kind of difference between Bob and Cam may be given a different theoretical description, but will be just as inevitable. With a degree theory, we might describe Bob and Cam as aiming at the right degree of truth with their differing degrees of belief in "Tek is tall", where their accuracy depends on how close that degree is to the actual degree. Their degrees of belief still differ from one another and almost certainly both come apart from genuine degrees of truth. With any illumination of degrees of belief and how vagueness impacts on our belief states, we must be able to accommodate this kind of difference between Bob and Cam. Smith's theory fails to do so as the degree of belief on that account is determined by degrees of truth across the various relevant possible worlds. For example, in an UFS, Bob and Cam-and everyone else-would be inappropriately assigned the same degree of belief in the vague proposition for the formula will equate degree of belief with degree of truth in the one world not ruled out. 
We might summarise that the problem here is with the role that the account attributes to objective degrees of truth in the story about subjective degrees of belief. This invites the reply that this seems to show too much as "it would apply just as much against the standard classical probabilist story about degrees of belief." 5 For the standard account of subjective probabilities (ignoring vagueness) also involves objective (classical) truth-values at different worlds, identifying the subjective probabilities with expected values across those worlds. But there is a crucial disanalogy here. Although the values in each relevant possible world are not a subjective matter, the determination of the extent to which each of those worlds contributes to the expected value $i s$ determined by subjective matters. Its contribution is not determined by its objective probability, but by its subjective probability, or the subject's judgement of the likelihood that it is the actual world. By contrast, the element corresponding to vagueness is determined by objective degree of truth. This can be seen in the VFS (vagueness-free situation), where (for the finite case), to get the subject's degree of belief in p, we sum the likelihood as judged by that subject of those worlds in which it is true. So, different subjects have different degrees of belief on this model. In an UFS, however, the subjectivity is lost. Suppose, for example, we are judging "Tek is tall" when Tek is standing in front of us and we are certain we are in full knowledge of his actual height etc. The subjective uncertainty is lacking here, so if Tek is borderline tall, my intermediate degree of belief is entirely due to vagueness. On Smith's account here, my degree of belief is equal to the actual degree of truth of the sentence and everyone else's degree of belief is determined as exactly the same.

So, the objection to Smith's generalisation to the case of degrees of truth is that to get a measure that reflects both subjective elements would need to factor in not just the extent to which a world is judged likely, but the degree to which the subject judges the proposition to be true in the world in question, not the objective value in that world.

Another way to bring out the objection here is to reflect on an appealing characterisation of degrees of belief to which Smith himself subscribes: "a degree of belief that $\mathrm{P}$ is a strength of tendency to act as if P" (2008, p. 230). Ramsey presented degrees of belief this way, where the belief that $\mathrm{p}$ may lead to radically different actions in different circumstances (varying, among other things, over the subject's desires and other beliefs). ${ }^{6}$ As Smith argues, the differing degrees among borderline cases can yield a corresponding difference in strength of tendency to act. For example, if I want an F, then I am more inclined to select $a$ than $b$ if $a$ and $b$ are borderline Fs but $a$ is $\mathrm{F}$ to a higher degree, (i.e. when I believe " $a$ is F" to a higher degree than I believe " $b$ is F"). We will consider this understanding of degrees of belief in terms of tendency to act below, but for now notice that what determines a subject's tendency to act will be a subjective state of the subject not the objective fact about degrees of truth (or, more accurately, the objective facts about degrees of truth across the range of situations that the subject hasn't ruled out). Again, there will be differences between the tendencies of different subjects to act, even if they agree on scenarios that are or are not ruled out and the likelihood of each.

\footnotetext{
5 Thanks to an anonymous referee for putting the point this way.

6 Ramsey (1931).
} 
Smith's attempt to provide a unified theory of degrees of belief due to uncertainty and degrees due to vagueness thus fails.

\section{Motivations for a unified treatment}

Why might we assume that we need a unified treatment of degrees of belief due to subjective uncertainty and degrees of belief due to vagueness? One general argument is captured in this quotation from Smith: "one simply can't have two different strengths of tendency to act as if P, in a given set of circumstances" (2008, p. 231). One might think this for several reasons. First it might be thought to be integral to the very notion in question. Smith, for example, says, "the notion of "strength of tendency to act as if $\mathrm{S}$ ' is inherently a final 'summing-up' notion: whatever complex factors go into [it] ... the tendency itself can have only one strength" (2014, p. 1031). But if it is inherent to that notion, maybe we should respond by thinking "so much the worse for that notion". It could be a confused notion that doesn't correlate to anything in our psychological states. I will argue that the situation with our beliefs and tendencies to act is sufficiently messy as to cast doubt on a single unified idea of tendency to act or degree of belief.

Such complexities are also brought out in relation to the second (closely related) reason to adopt a unified notion of degree of belief in the light of both subjective uncertainty and vagueness, which turns on the relation between the degree of belief (or tendency to act) and actual action. Smith uses a number of illustrative examples to substantiate such a reason. For example, our attitude to "Fido is dangerous" might be affected by both vagueness and uncertainty, but, claims Smith, "one cannot both tend strongly to act as if Fido is dangerous and tend weakly to act as if Fido is dangerous". He points out that in a particular circumstance (when he barks, say), you "will do some particular thing ... one cannot both back away slowly and run screaming (at the same time)". It might be argued, then, that there is a single story about a subject's actual action, so there must be a single story about that subject's strength of tendencies to act from which this is an upshot. This is bound up with a commitment that Smith describes as a "transparent relationship between tendencies to act and the way one actually acts" (2008, p. 231). For example, he complains that an alternative to his view "threatens to make it impossible for us ever to know (even roughly) someone's degree(s) of belief in a given proposition" (p. 495). But such a transparency is hard to sustain given that tendencies to act do not always manifest themselves in actual action and are compatible with many different actions.

Smith acknowledges the possibility of different factors contributing to the tendency to act, where those factors may have different weights, though, as we've seen, he maintains that they contribute to a "summing-up" strength of tendency. But the unity of action (in the sense explained) does not give grounds for commitment to a unique and unified strength of tendency to act.

Perhaps there is no single uniform story to be told here. The description of one's tendencies to act - and correspondingly the account of one's degrees of belief-is far messier than on a picture like the above and the notion of tendency to act threatens to be too crude to accommodate the fine-grained structure of degrees such a story 
requires. Let's consider some examples. Suppose you are choosing someone for the basketball team and you want someone tall. Faced with two borderline tall men, other things being equal, you are more likely to choose $\mathrm{x}$ over $\mathrm{y}$ if " $\mathrm{x}$ is tall" is true to a higher degree than " $y$ is tall". But we can imagine a context where a parallel set-up-a desire for an $\mathrm{x}$ which is $\mathrm{F}$ and the offer of borderline cases of $\mathrm{F}$ - where the tendencies form a different pattern differing from the simplest one envisaged. To adapt a case of Edgington's (1996), I might have a desire for coffee, but have no tendency at all to reach for something on the borderline between coffee and tea and I might well lack an increasing tendency to reach for something the further through the borderline cases towards the coffee end it is. Or in another case, I may have some tendency to reach for a borderline $\mathrm{F}$ among the end of the borderline-Fs closest to $\mathrm{F}$, where this drops off swiftly towards the middle of those borderline cases.

We might deal with some of these cases by re-describing the desire. Maybe my desire for coffee should really be described as a desire for something that is definitely coffee, which would be compatible with my revulsion towards borderline coffee-tea (and even cases that are nearly definitely coffee). ${ }^{7}$ And that may be contrasted with a case where I want an $\mathrm{F}$ and a borderline $\mathrm{F}$ will fulfil that desire to the degree to which it counts as F. But even if this works in this coffee case, those cases with a more complicated pattern of behaviour over the borderline cases will still be problematic. For example, consider cases where there is a less gradual drop-off in the extent to which my desire is satisfied by borderline Fs, corresponding neither to the degree of " $\mathrm{x}$ is definitely F" nor of " $\mathrm{x}$ is F". Such a pattern can be perfectly natural and rational.

We could try to avoid these difficulties by restricting the cases that allow us to measure degrees of beliefs in terms of tendency to act, so that we consider only those cases that do follow the simple pattern that Smith illustrates, where, alongside the subject's desire for an F, it seems right to say that they desire something to the extent that it is F. Smith, for example, simplifies his case in which the subject has a preference for someone tall, by expressing their preference in terms of "the taller the better" (Smith 2014, p. 1035). But with that description of the case, the subject's degrees of belief in " $x$ is tall" aren't needed to play a role. The best explanation of their actions won't use the vague notion of "tall" at all, but will appeal to the subject's beliefs involving "taller than" or more specific classifications of heights. They prefer $a$ to $b$ because they believe that $a$ is taller than $b$, not because they believe " $a$ is tall" to a greater degree than they believe " $b$ is tall". So, a restriction to such cases will not help illuminate degrees of belief surrounding borderline cases at all.

There is always bound to be a rich variety in kinds of cases where the subject's recognition of something as a borderline case effects the way they act and their tendency to act in certain ways. This is not, on its own, provide a knock-down argument against offering a unified account such as Smith's, and the examples he discusses are intended merely as a relatively simple illustration. But, I suggest that acknowledging the complexity of cases beyond these counts against the attempt to ground a unified

\footnotetext{
7 In a similar way, Smith considers a case where the subject wants someone very tall rather than just someone tall (2010, p. 498).
} 
and universal notion of "tendency to act" to identify with the desired notion of degrees of belief. 8

In modelling subjective probabilities, the use of betting odds is a powerful method that allows us to make sense of the idea that our subject has finely grained degrees of belief. We can compare her degree of belief in $\mathrm{p}$ and $\mathrm{q}$ by determining for which she would take the highest odds. And when we're not bombarding our subject with questions about how she would bet, we can settle for the assumption that she has particular dispositions to answer such questions. ${ }^{9}$ This device won't transfer easily to degrees of belief in the light of vagueness, though. Smith, for example, says, "one should not bet at all on a proposition $S$ unless one is in a vagueness-free situation with respect to $S$ " (p. 503); he argues that you are irrational if you do bet, for the bet will go undecided. This, then, would block off the main well-honed resources for getting a grip on fine-grained differences between degrees of belief. There may be ways round these concerns and some (artificial) way to make sense of ascription of betting behaviour despite this. Indeed, Smith (2014, pp. 1037-1038) offers an argument to show that in following the pattern of degrees of belief sustained in his theory, a subject will not be subject to a Dutch Book. The apparent tension with both regarding betting as irrational and giving this role in the theory is resolved by appeal to two different notions of betting. ${ }^{10}$ On the familiar notion whereby winning anything on a bet on $\mathrm{P}$ requires $\mathrm{P}$ to be true, betting is irrational. On the alternative notion, partial winnings are possible and determined by the extent to which $\mathrm{P}$ is true. This latter notion is an unusual and, in practice unworkable idea of betting (there could be no settling on the outcome). Whereas appeal to the former idea of betting might have some claim to a role in illuminating the tendencies to act of our subjects who understand such an idea, the new, artificial notion of betting is not well suited to such a role.

As Smith says (pp. 504-505), we can make sense of tendency to act without reference to betting behaviour; but it isn't clear how we can get a numerical scale (or equivalent) out of the kinds of crude measures proposed. Such methods include comparative measures of degrees of belief in different propositions, but these methods will be very limited and often questionable. For example, as we have seen, Smith and others use the idea of asking the subject to choose an F. If S chooses x over $y$, we can conclude that $S$ believes " $\mathrm{x}$ is $\mathrm{F}$ " to a higher degree than " $y$ is F", all things being equal (a not insignificant qualification). To compare S's belief in " $x$ is $F$ " and in " $y$ is G" we would have to set $S$ the task of assuming they want an F or a $G$, with no preference, then answering which would they choose. This would be yield some very puzzling questions for the subject, such as, "if you wanted a red ball or a big rabbit and wanted those things to the same extent, what would you choose between this (bor-

\footnotetext{
8 E.g. the simplified discussion of the basketball case in Smith (2014) is used to argue that his theory delivers values for degree of belief that $S$ that "match the intuitive strengths of the agent's tendencies to act as if S", but I question the assumption that there are such intuitive strengths when $\mathrm{S}$ is recognisably borderline.

9 This is, of course, very artificial and may be questionable in various ways; I won't enter into such doubts here. I claim that degrees of belief associated with vagueness are more problematic than those associated with uncertainty; if the latter are problematic too, those problems are very likely to be additional problems for the former case as well.

10 See Smith (2008, p. 503, footnote 19) where he distinguishes these two notions of "bet"; see also (2014, p. 1038) on the new notion of betting.
} 
derline red) ball and this (borderline big) rabbit?" If we force our subject to choose, we can't assume that we're uncovering the structure of their mental states rather than witnessing the results of an arbitrary choice between options that strike the subject as equally good or not really comparable.

Could we see this as purely an epistemic problem of finding out what the subject's degrees of belief are, maintaining that their structure neatly mirrors the structure of the real numbers between 0 and 1 as the theory requires, even if we have extremely limited grasp on the instantiation of that structure? I suggest that this position is very unappealing as the grounds for postulating this structure are weak. Put in Smith's own terms, this response and the imposition of a structure we can't detect by its manifestations is very much against the spirit of his commitment to a "transparent relationship" between tendencies to act (which, recall are the essence of degrees of belief) and the way one actually acts.

In Sect. 1, I summarised three options for considering degrees of belief in the light of both subjective uncertainty and vagueness. First, the option I have rejected here was to offer a unified account of the two phenomena, resulting in a single value for one's degree of belief, reflecting contributions from both components. The second approach was to give separate accounts of two types of degrees of belief and treat subjects as sometimes having a different degree of belief of each kind in the same proposition at the same time. I will not say much about that option here, treating it as a prior question whether and how we should or can develop an account of degrees of belief arising from vagueness. The third option denies that such an account is viable and I say a little more about this option at the end of the paper. First, I briefly ask whether we should regard an account such as Smith's as an idealisation, and whether that would solve problems raised.

\section{Expected degree of belief as an idealisation?}

If we conclude from the above that Smith's expected degrees of belief do not provide a good account of actual degrees of belief, can they still function as a useful idealisation in some form or other? One suggestion might be that expected degrees of belief should be seen as defining a notion of degree of belief instead. Perhaps we should give up on modelling subjects' actual messy states of mind and use a theoretical notion of degrees of belief defined in Smith's terms. This, note, would be a very different role with severe limitations. As explained above, it could not be related to the tendency to act in the way Smith hopes and, similarly, will surely have limited use in predicting behaviour. In combining subjective probability with objective degrees of truth, we would have neither a subjective account we can use to explain or predict behaviour, nor an objective account for use in the semantics.

Could it provide directions for adapting degrees of belief when vagueness as well as uncertainty is in play, however? For example, it could then help me work out to what degree I should believe that the son of my two old friends is tall, given my judgements of the likelihood that he is of various heights and the semantics of the vague "tall". It would thereby give me a "summing-up" figure for my degree of belief in the light of both the vagueness and the uncertainty, and it might be thought that this figure is what 
should guide my action. But, whether it should indeed guide my action will depend on all sorts of specifics or elements of contexts. For example, Smith himself will have to add that my action in taking bets shouldn't be governed by the figure for my degree of belief that this method provides, since he maintains that we shouldn't bet in circumstances of partial belief resulting from vagueness. By contrast, the same degree of belief in a vagueness-free situation should determine appropriate betting behaviour, so the "summing-up" value is of little use here without knowledge of the components that went into it. Outside betting contexts, there will be other differences in appropriate actions according to whether vagueness or uncertainty unrelated to vagueness are at issue. This, then, is another role to which expected degrees of belief are unsuited. ${ }^{11}$

\section{Rejecting the focus on degrees in modelling and understanding vagueness}

If our attitudes to borderline cases aren't best presented by an account of degrees of belief along the lines of Smith's, how can we best consider those attitudes and how, if at all, does this relate to the choice between different theories of vagueness? In many cases where $a$ is recognisably a borderline case of F, our attitude to " $a$ is F" might be reflected in a common way in which we'd respond verbally to the question whether $a$ is F when it is borderline, namely to say that we don't know, or that it neither is nor isn't or to avoid answering, perhaps by saying instead that $a$ is F-ish. An attitude producing this kind of verbal behaviour may not be well represented by the assignment of some exact degree of belief.

I would argue that we should not expect one's theory of vagueness to deliver an account of what psychological states we have (or even should have) in the face of borderline cases. Adopting some particular theory should allow us to recognise that subjects are alert to the difference between definite Fs and borderline Fs, but should be compatible with a range of attitudes to borderline cases, so that, when $a$ is borderline $\mathrm{F}$, we will sometimes count as not believing that $a$ is F, sometimes count as partially believing it, sometimes count as being uncertain about it (where this is not well captured by a degree of uncertainty) and sometimes, but only sometimes, count as believing it to some relatively exact degree.

This is an approach that could be adopted alongside a commitment to any of a range of different theories of vagueness (or, indeed, an agnosticism between them). Consider, for example, the supervaluationist theory of vagueness according to which a sentence is true (false) iff it is true (false) on all precisifications of it, making a borderline case predication neither true nor false. ${ }^{12}$ That classification is compatible with the reasonableness of the described range of attitudes to the predication-expression of ignorance or uncertainty, or its redescription in hedged terms like "F-ish". As is well known, it also yields an appealing story about our attitude to certain compound sentences known as penumbral connections. For example, a disjunction such as "this is

\footnotetext{
11 Indeed, this problem for the "summing up" value arises whether or not we take the account as providing an idealisation. The differing behaviour in betting situations shows that one's behaviour or tendency to act is not well represented by a figure that does not distinguish between subjective uncertainty and vagueness cases. 12 See Fine (1975) and Keefe (2000).
} 
red or orange" said of a patch of the colour on the borderline between red and orange, can count as true, reflecting the fact that we are strongly inclined to assent to it. On Smith's view, by contrast, it can only be as true as the most true disjunct, and so our degree of belief in it should be no higher than that on his view.

Now, Supervaluationism can be supplemented with a story about degrees as measures over valuations, so that $a$ counts as $\mathrm{F}$ to degree $\mathrm{d}$ if the proportion of precisifications in which $a$ is $\mathrm{F}$ is d. ${ }^{13}$ The degrees captured this way are not degrees of truth; a sentence less than degree 1 true is neither true nor false without being true to an intermediate degree. It may sometimes help model our belief states using these degrees and may sometimes help us judge how to adapt our beliefs, but the role of degrees should be seen in this instrumentalist way. The relation between this model of degrees and our actual psychological states needn't be straightforward or uniform.

I suggest that there is not even a unified account of degrees of belief for cases involving vagueness in the absence of the other kind of uncertainty, so that it is not beneficial to seek a degree-theoretic account combining the two.

It may well help, in pursuing a picture about our subjective states, to keep in mind different theories of vagueness. But we should not expect a neat theory about degrees of belief to be yielded by the chosen theory of the semantics of vagueness. In particular, the similarity between the semantic machinery of the degree theory of vagueness and degrees of belief does not, it turns out, make such a theory particularly well suited to illuminating degrees of belief and more specifically, Smith's interesting theory along these lines does not work.

Acknowledgements I'm very grateful to two anonymous referees and the editor of this special issue for useful comments on earlier drafts of this paper.

Open Access This article is distributed under the terms of the Creative Commons Attribution 4.0 International License (http://creativecommons.org/licenses/by/4.0/), which permits unrestricted use, distribution, and reproduction in any medium, provided you give appropriate credit to the original author(s) and the source, provide a link to the Creative Commons license, and indicate if changes were made.

\section{References}

Edgington, D. (1996). Vagueness by degrees. In R. Keefe \& P. Smith (Eds.), Vagueness: A reader. Cambridge, MA: MIT Press.

Fine, K. (1975). Vagueness, truth and logic. Synthese, 30, 265-300.

Kamp, J. A. W. (1975). Two theories about adjectives. In E. L. Keenan (Ed.), Formal semantics of natural language. Cambridge: Cambridge University press.

Lewis, D. (1970). General semantics. Synthese, 22, 18-67.

Keefe, R. (2000). Theories of vagueness. Cambridge: Cambridge University Press.

Ramsey, F. P. (1931). Truth and probability. In R. B. Braithwaite (Ed.), The foundations of mathematics and other logical essays. London: Routledge.

Schiffer, S. (2000). Vagueness and partial belief. Philosophical Issues, 10, 220-257.

Smith, N. J. J. (2008). Vagueness and degrees of truth. Oxford: Oxford University Press.

Smith, N. J. J. (2010). Degree of belief is expected truth value. In R. Dietz \& S. Moruzzi (Eds.), Cuts and clouds: Vagueness, its nature and its logic. Oxford: Oxford University Press.

Smith, N. J. J. (2014). Vagueness, uncertainty and degrees of belief: two kinds of indeterminacy-One kind of credence. Erkenntnis, 79, 1-18.

13 See Kamp (1975), Lewis (1970) and Keefe (2000, pp. 171-173). 